\title{
Rapid Taste Responses in the Gustatory Cortex during Licking
}

\author{
Jennifer R. Stapleton, ${ }^{1}$ Michael L. Lavine, ${ }^{2}$ Robert L. Wolpert, ${ }^{2}$ Miguel A. L. Nicolelis, ${ }^{1,3,4,5}$ and Sidney A. Simon ${ }^{1,3,5}$ \\ ${ }^{1}$ Department of Neurobiology, ${ }^{2}$ Institute of Statistics and Decision Sciences, ${ }^{3}$ Department of Biomedical Engineering, ${ }^{4}$ Department of Psychological and \\ Brain Sciences, and ${ }^{5}$ Center for Neuroengineering, Duke University, Durham, North Carolina 27710
}

Rapid tastant detection is necessary to prevent the ingestion of potentially poisonous compounds. Behavioral studies have shown that rats can identify tastants in $\sim 200 \mathrm{~ms}$, although the electrophysiological correlates for fast tastant detection have not been identified. For this reason, we investigated whether neurons in the primary gustatory cortex $(\mathrm{GC})$, a cortical area necessary for tastant identification and discrimination, contain sufficient information in a single lick cycle, or $\sim 150 \mathrm{~ms}$, to distinguish between tastants at different concentrations. This was achieved by recording neural activity in GC while rats licked four times without a liquid reward, and then, on the fifth lick, received a tastant (FR5 schedule). We found that $34 \%$ ( 61 of 178) of GC units were chemosensitive. The remaining neurons were activated during some phase of the licking cycle, discriminated between reinforced and unreinforced licks, or processed task-related information. Chemosensory neurons exhibited a latency of 70-120 ms depending on concentration, and a temporally precise phasic response that returned to baseline in tens of milliseconds. Tastant-responsive neurons were broadly tuned and responded to increasing tastant concentrations by either increasing or decreasing their firing rates. In addition, some responses were only evoked at intermediate tastant concentrations. In summary, these results suggest that the gustatory cortex is capable of processing multimodal information on a rapid timescale and provide the physiological basis by which animals may discriminate between tastants during a single lick cycle.

Key words: gustatory cortex; taste; generalized linear model; licking; electrophysiology; fixed ratio schedule

\section{Introduction}

A primary function of the gustatory system is to analyze the chemosensory, somatosensory, and hedonic properties of potential food sources to determine whether they should be ingested. In analyzing neurophysiological responses from behaving animals, the interpretation of chemosensory signals may be confounded with these multisensory and motivational contributions. Many of these influences can be controlled by using anesthetized animals, because in such studies there is no motivational component, the tongue is immobilized, and there is continuous stimulus flow, which can cause somatosensory system adaptation to mechanical and thermal input. However, in freely behaving, motivated animals, the somatosensory and gustatory systems are intimately coupled and contribute to stimulus coding (Zeigler et al., 1984; Berridge and Fentress, 1985; Todrank and Bartoshuk, 1991; CerfDucastel et al., 2001; Katz et al., 2002; de Araujo and Rolls, 2004; Small et al., 2004; Gutierrez et al., 2006). Previous electrophysiological recordings from the rat primary gustatory cortex (GC), a cortical area necessary for tastant identification and discrimination (Yamamoto et al., 1980, 1984; Ogawa et al., 1992), have been performed in rats permitted to freely lick to receive tastants (Yamamoto et al., 1988, 1989; Bahar et al., 2004). However, in

Received Jan. 9, 2006; revised March 10, 2006; accepted March 11, 2006

This work was supported in part by National Institutes of Health Grant DC-01065, Philip Morris International, and Philip Morris USA. We thank Ranier Gutierrez, Patrick Fisher, Jim Meloy, and Jessica Lynch.

Correspondence should be addressed to Jennifer R. Stapleton, Department of Neurobiology, Duke University, Durham, NC 27710. E-mail: stapleton@neuro.duke.edu.

DOI:10.1523/JNEUROSCI.0092-06.2006

Copyright $\odot 2006$ Society for Neuroscience $\quad$ 0270-6474/06/264126-13\$15.00/0 these circumstances, studies of gustatory coding may have been confounded by somatosensory input driven by rhythmic licking.

To separate the neural activity elicited by the tastant itself from the activity elicited by the sensory and motor features of licking, rats were trained to receive tastants on a fixed ratio schedule (FR5) while GC responses were recorded from implanted microelectrode bundles. In this testing schedule, rats licked a dry sipper spout four times and received a tastant only on the fifth lick. Therefore, the responses to licking alone can be compared with those elicited by tastants. In addition, trained rats have been shown to identify tastants in $\sim 200 \mathrm{~ms}$ (Halpern and Tapper, 1971), which is about the time frame of one lick cycle (Spector et al., 1996; Travers et al., 1997), but the electrophysiological correlates of rapid tastant identification have yet to be identified. We chose to analyze such multimodal, temporally oscillating responses with a generalized linear model ( $\mathrm{glm})$. In this approach, the logarithm of the Poisson firing rate for each neuron is modeled as a linear combination of explanatory variables, such as indicators of the various tastant/concentration combinations and of the time lapse since the lick onset (McCullagh and Nelder, 1989; Grimmett and Stirzaker, 2001). With the glm, we have successfully distinguished between reinforced and unreinforced licks, separated tastants and their concentrations, and measured the evolution of the chemosensory response over the course of a single lick. These results suggest that tastant-evoked responses in the rat gustatory cortex process multimodal information on a rapid timescale and provide the physiological basis by which animals might discriminate between tastants during a single lick. 


\section{Materials and Methods}

Subjects. Male Long-Evans rats $(n=5)$ were obtained from Harlan Bioproducts for Science (Indianapolis, IN). Before surgery, all subjects weighed $300-450 \mathrm{~g}$. The rats were housed individually in Plexiglas cages and were maintained on a $12 \mathrm{~h}$ light/dark schedule, with experiments performed in the light portion of the cycle. The subjects were permitted to recover from surgery for 2 weeks, after which they were placed on a $20 \mathrm{~h}$ water deprivation schedule. In addition to the water available during each 2 or $3 \mathrm{~h}$ test session, subjects were also permitted $1 \mathrm{~h}$ of access to water in their home cages. Purina rat chow was available ad libitum.

All protocols were approved by the Duke University Institutional Animal Care and Use Committee.

Surgery. Rats were anesthetized initially with a 5\% halothane/air mix and then with a $50 \mathrm{mg} / \mathrm{kg}$ intraperitoneal injection of sodium pentobarbital. They were placed in a standard stereotaxic frame, and the scalp and overlying fascia were retracted. Four stainless-steel screws, one per cranial plate, were inserted into the skull, and ground wires for the electrodes were wrapped around the screws. Moveable electrode bundles (16 $15-\mu \mathrm{m}$ tungsten microwires per cannula shaft) were implanted bilaterally above GC ( $1.3 \mathrm{~mm}$ anterior, $5.2 \mathrm{~mm}$ lateral, and $4.7 \mathrm{~mm}$ horizontal from bregma) (Kosar et al., 1986; Katz et al., 2001, 2002), and then dental acrylic was applied to seal the exposed skull surface and electrode bundles. After 1 week of recovery, the electrodes were lowered $100 \mu \mathrm{m}$ per day until reaching the gustatory cortex (see Fig. 1).

General electrophysiology. Once the electrodes were located in GC, recording commenced. When the signals at the current location had degraded, the electrodes were lowered further in GC in $100 \mu \mathrm{m}$ increments.

Throughout the experiment, neural activity was recorded continuously. Differential recordings were fed into a parallel processor that simultaneously digitized the analog signals from several channels at $40 \mathrm{kHz}$ (Plexon, Dallas, TX). Discriminable action potentials with a signal/noise ratio $\geq 3: 1$ were isolated on-line from each channel through the use of template matching in conjunction with three-dimensional principal component analysis. The refractory period for single units was established as $2 \mathrm{~ms}$. Time-stamped records of stimulus onsets, spiking events, and all spike waveforms were stored digitally for additional off-line sorting.

Behavioral apparatus. All testing occurred in Med Associates (St. Albans, VT) operant chambers that were located within sound attenuating boxes. At one end of the operant chamber was a rectangular recess in the wall $5 \mathrm{~cm}$ from the floor and $5 \times 5 \times 2.5 \mathrm{~cm}$ in dimension. This portal housed an infrared Med Associates lickometer that was positioned directly in front of the lick tube. The lick tube itself was comprised of a bundle of 1220 -gauge stainless-steel tubes cemented together in a larger steel tube (inner diameter, $7.5 \mathrm{~mm}$ ), and the smaller tubes terminated at the end of the large tube such that there was no dead space in which tastants might mix.

Each taste solution was contained within 1 of 1250 -ml chromatography columns (Kontes Flex-Columns; Fisher Scientific, Hampton, NH), and the system was maintained under $\sim 8$ psi of air or nitrogen. Computer-controlled solenoids (Parker Hannifin Corporation, Fairfield, NJ) regulated the flow of fluid from the columns through tubing connected to the lick spout. The columns and solenoids were housed outside the sound-attenuating chambers. Within $10 \mathrm{~ms}$ after a lick was detected because of the breaking of the infrared beam, one of the valves opened and delivered $\sim 50 \mu \mathrm{l}$ of fluid.

Behavioral testing. Water-deprived rats were tested using a brief access paradigm. In a given testing session, subjects were presented with a set of 5-12 tastants at different concentrations. These tastants included the following: sucrose, monosodium glutamate (MSG), and $\mathrm{NaCl}(0.025$, $0.075,0.1$, and $0.3 \mathrm{M})$; quinine $\mathrm{HCl}(0.0001$ and $0.0003 \mathrm{M})$; citric acid $(0.005$ and $0.01 \mathrm{M})$; and distilled water. The concentrations of sucrose and MSG were all suprathreshold and were approximately matched for perceptual intensity. The highest concentrations of each were within the palatability limits for rats (Delay et al., 2000; Stapleton et al., 2002). The concentrations for $\mathrm{NaCl}$ and quinine were similarly chosen, although, compared with water, the concentrations of quinine tested were less palatable (Spector and St. John, 1998). The concentrations for citric acid were chosen to approximately match previous electrophysiological studies (Plata-Salaman et al., 1995; Katz et al., 2001, 2002). All chemicals were obtained from Sigma (St. Louis, MO) and were reagent grade. In addition to being used as a rinse, water was also considered to be a tastant (de Araujo et al., 2003). This is because the responses it evoked differed from the unreinforced licks and because its activity could be discriminated from the activities elicited by the other tastants (see Figs. 3, 4, 7-11). In addition, taste receptor cells respond to changes in volume such as may occur in the presence of water (Lyall et al., 1999, 2005).

Most of the experiments were performed with a fixed ratio schedule (FR5) in which water-deprived rats were trained to lick a dry sipper spout from which $50 \mu \mathrm{l}$ of a given tastant were delivered every fifth lick. A trial consisted of eight deliveries of a particular tastant, whereupon the trial ended and the subject entered a 5-10 s intertrial interval (ITI). At the end of this ITI, one or two water "washouts" were delivered (50-100 $\mu \mathrm{l})$. This washout was followed by a second 5-10 s ITI period, whereupon a subsequent trial with a different tastant began. The choice of tastant to be delivered on each trial was randomized without replacement using a Latin square protocol. Within a given testing session, each tastant was presented in multiple trials (3-8) for a total of 24-64 deliveries. The tubes were randomly assigned to contain different tastants on each day of testing to prevent the rats from anticipating the delivery of certain tastants based on possible auditory cues from the solenoids.

We used $50 \mu$ l of fluid as a follow up to previous work in our laboratory (Katz et al., 2001, 2002), where responses in rat GC were recorded after the delivery of $50 \mu \mathrm{l}$ of tastants through intraoral cannulas. Another reason for using this volume is that we also wanted to stimulate a relatively large region of the tongue and palate.

Experiments were also performed using a variable ratio schedule (VR5) to test for possible effects of tastant expectation. After the subjects had become accustomed to the FR5 schedule, they were switched to a VR5 schedule. In this schedule, four to six licks were required for the tastant to be delivered, with an average criterion of five licks. The number of licks required was randomized without replacement. This permitted the activities of the reinforced licks for each schedule to be compared to assess whether chemical tuning remained stable. If the response profile remained stable, then this implied that the evoked responses were indeed strictly chemosensory and were not confounded by the effects of expectation.

Data analysis. For hedonically positive tastants, rats lick at $\sim 6-8 \mathrm{~Hz}$ (Wiesenfeld et al., 1977; Spector and St. John, 1998; Gutierrez et al., 2006) or about every $150 \mathrm{~ms}$. For this reason, a $150 \mathrm{~ms}$ window was taken from the time of each tastant delivery. This window was further divided into 10 15 -ms bins. Empirically, we found a $15 \mathrm{~ms}$ bin to contain a sufficient number of spikes such that the spike rate could be modeled reasonably well (see below).

A second 150 ms window was taken starting from the time of the third unreinforced lick in the FR5 cycle and these spikes were similarly placed into 1015 -ms bins. The spike train for this unreinforced lick was chosen to represent the background activity before tastant administration because the third lick was sufficiently distanced from the time of the tastant delivery such that overlap between the two $150 \mathrm{~ms}$ windows would not occur. Because the interval between some licks can be shorter or longer than $150 \mathrm{~ms}$, some of the binned data will contain or miss spikes from the subsequent lick. However, this is relatively rare because most chemosensitive responses were transient, had an onset latency of $\sim 50 \mathrm{~ms}$, and finished before the next lick, and because the glm accurately represented the firing rates (see Figs. 2, 3, 7-12). Consequently, we believe the use of a standard $150 \mathrm{~ms}$ interval is justified. We also considered normalizing the number of spikes that occurred during each interlick interval by the length of the interval, but this would have produced fractional spike counts that could not have been modeled with a Poisson distribution.

The binned spike trains were tagged with unique identification numbers representing the bin number in the window and imported into and analyzed in the statistical program " $R$ " (www.R-project.org). The spike count $X$ for each bin was modeled as $X \sim$ Poisson $(\lambda)$; in other words,

$$
P[X=k \mid \lambda]=\frac{\lambda^{k} e^{-\lambda}}{k !},
$$


where $\lambda$ may depend on the particular neuron, the bin, reinforcement, trial number, and delivery number. The generalized linear model says the following:

$$
\ln \lambda=\beta_{0}+X \beta
$$

where the columns of the matrix $X$ contain the covariates, which include indicator variables for reinforcement, concentrations of each tastant, bin number, trial number, tastant delivery number within each trial, and possibly their interactions. The parameter vector $\beta$ is the relative contribution of each covariate to the fit of the data, and $\beta_{0}$ is the intercept.

The model was fitted separately for each neuron. Terms for main effects and interactions were included according to the amount of deviance they explain. [The more complex of two nested models will always have a higher likelihood, but it is only preferred to the simpler model if the reduction in deviance (twice the negative natural logarithm of the likelihood, a generalization of the sum-of-squared-errors from normaldistribution regression models) is large compared with the increase in model dimension.] The significance of each term was assessed according to the $\chi^{2}$ distribution. Neurons were classified as chemosensory if they could both discriminate between reinforced and unreinforced licks and between tastants [i.e., the coefficients of the model that corresponded to reinforcement and to the tastants were required to be significant (all values of $p<0.05)$ ].

To test the ability of the model to fit the data accurately, a $90 \%$ predictive interval was constructed around each of the fitted values, and the number of corresponding observed values that fell within this interval was calculated. Ideally, $90 \%$ of the observed data should be contained within the $90 \%$ predictive interval. We found that the coverage probabilities were reasonable, being $\sim 86 \%$ (see Results).

We also tested whether bin sizes other than $15 \mathrm{~ms}$ would account for a greater percentage of the deviance in the data sets. Twenty chemosensory neurons were randomly chosen from three animals, and the spike trains were modeled with bin sizes of 30, 75, and $150 \mathrm{~ms}$. For each neuron, the likelihood ratio test was used to compare these models with the model based on $15 \mathrm{~ms}$ bins. In all cases, the model based on the $15 \mathrm{~ms}$ bins captured significantly more deviance than models constructed with a larger bin size. In particular, relative to the model based on $15 \mathrm{~ms}$ bins, the models based on bins of 30, 75, and $150 \mathrm{~ms}$ captured an average 93.4, 87.1 , and $81.8 \%$ of the total deviance. These percentages were derived by taking the amount of deviance for which each model accounted and dividing it by the amount of deviance accounted by the model based on $15 \mathrm{~ms}$ bins. Relative to the first ( $15 \mathrm{~ms}$ ) model, the performance of the models for 30, 75, and $150 \mathrm{~ms}$ bin sizes significantly decreased as follows: values of $p<0.01$ (with 12 of 20 neurons differing significantly), values of $p<0.01$ (for all 20 neurons) and values of $p<0.004$ (for all 20 neurons), respectively.

To test for the effects of expectation of the tastant in the FR5 schedule, the data were binned into $15 \mathrm{~ms}$ increments and the second and fourth unreinforced licks were compared. These particular licks were chosen because they were well separated from the tastant. In addition, these licks might differ if the rats could track the number of licks necessary for tastant delivery (Meck, 2003). As a second test for expectation, the reinforced licks obtained under the FR5 and VR5 schedules were compared. It is possible that the spike trains recorded during reinforced licks in the FR5 schedule are a composite of tastant-related information and signals arising from expectation for receiving a tastant on a particular lick. In contrast, spike trains recorded during the VR5 schedule should contain chemosensory information without the expectation for reinforcement on a particular lick. If the chemical tuning profiles of neurons do not differ under the FR5 and VR5 schedules, then the effects of expectation would not confound our identification of chemosensory signals.

Calculations of entropy, time to peak, and onset and offset times. The entropy $(H)$, or breath of tuning, was calculated for each neuron by means of the following (Smith and Travers, 1979):

$$
H=-k^{\prime} \sum_{i=1}^{n} p_{i} \log p_{i}
$$

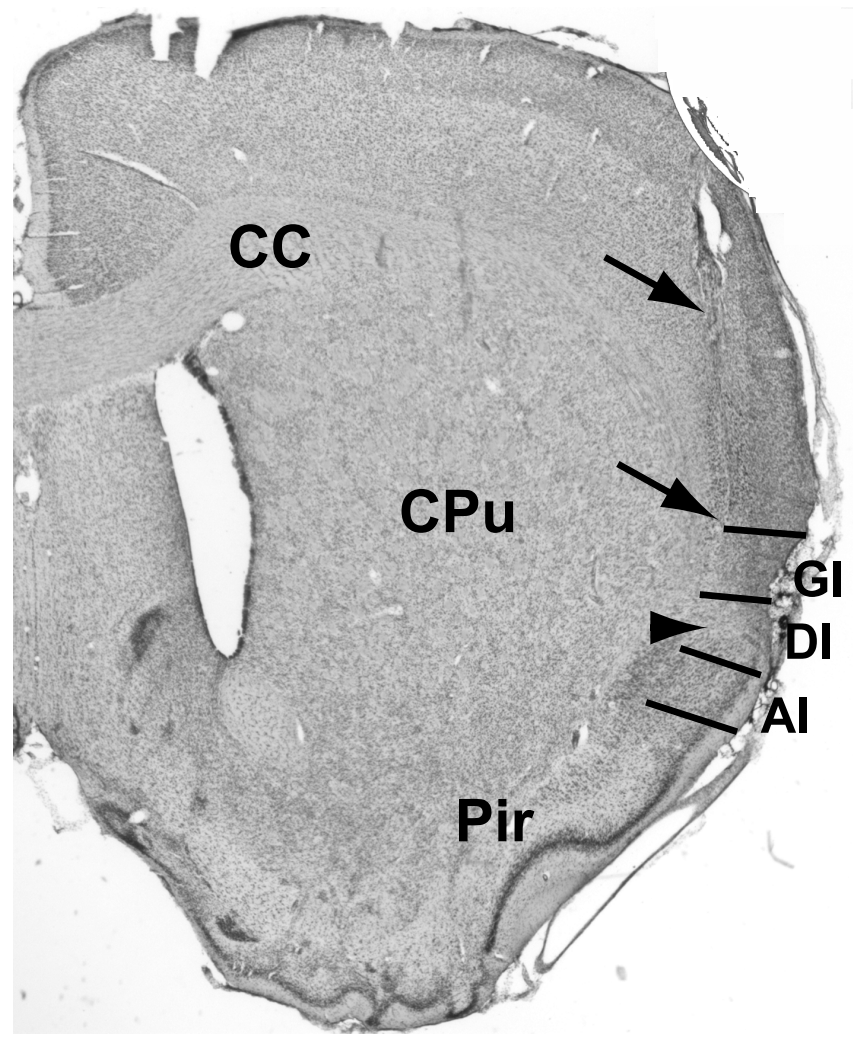

Figure 1. Histology. Shown is a 50- $\mu$ m-thick coronal section that was taken through the gustatory cortex and stained with cresyl violet. The cannula track is clearly visible and is indicated by two arrows. The terminations of the electrodes in dysgranular cortex are marked by an arrowhead. CC, Corpus callosum; Pir, piriform cortex; $\mathrm{CPu}$, caudate-putamen; $\mathrm{Gl}$, granular insular cortex; DI, dysgranular insular cortex; Al, agranular insular cortex.

where $H$ is the entropy and possesses a maximal value of 1 when a neuron responds equally to all $n$ tastants. The proportional response $p_{i}$, evoked by each tastant, is expressed as a ratio of the firing rate for a given tastant over the $150 \mathrm{~ms}$ window normalized by the sum of the firing rates obtained for all tastants. In the case in which a neuron responds equally to all tastants, $p_{i}=1 / n$, with $i$ referring to the $i$ th tastant, $n$ as the number of tastants, and $k^{\prime}$ as the scaling constant such that the maximum value that $H$ can attain is 1 . (In the case in which a neuron responds equally well to all $n$ tastants, $k=\frac{1}{\log n}$.) For three, four, and five tastants, $k^{\prime}=2.096$, 1.661 , and 1.431 , respectively. For each neuron, $H$ was calculated using the concentration for each tastant that evoked the maximal response. Hence, each tastant was represented once in the calculation.

For each tastant, the peak response times were defined as the time at which the maximum evoked response occurred in the peristimulus time histogram (PSTH). These data were then averaged across neurons to identify average peak firing rates and times for each tastant.

All data are reported as mean \pm SEMs.

Histology. After completion of the experiments, the rats were deeply anesthetized with an intraperitoneal injection of Nembutal $(150 \mathrm{mg} / \mathrm{kg})$ and perfused transcardially with $120 \mathrm{cc}$ of PBS followed by $120 \mathrm{cc}$ of formalin (10\%). Brains were removed and stored in a sucrose/formalin solution $(30 \%$ sucrose $\mathrm{w} / \mathrm{v})$ for $24 \mathrm{~h}$ of postfixation. The tissue was sectioned through GC in $50 \mu \mathrm{m}$ coronal slices and stained with cresyl violet to visualize cell bodies. This is sufficient to visualize electrode tracks, which were subsequently mapped to verify placement.

\section{Results}

\section{Histological results}

Electrode placement in GC was verified for all implanted rats. Figure 1 depicts a coronal section through GC in which the area 
Table 1. Characteristics of chemosensory GC neurons

\begin{tabular}{|c|c|c|c|c|}
\hline \multirow[b]{2}{*}{$\begin{array}{l}\text { Chemosensory } \\
\text { neurons }(n=61)\end{array}$} & \multicolumn{2}{|l|}{ Evoked responses } & \multicolumn{2}{|c|}{ Background responses } \\
\hline & $\begin{array}{l}\text { Max firing rate } \\
(\mathrm{sp} / \mathrm{s})\end{array}$ & $\begin{array}{l}\text { Time of max } \\
\text { firing rate (ms) }\end{array}$ & $\begin{array}{l}\text { Max firing rate } \\
(\mathrm{sp} / \mathrm{s})\end{array}$ & $\begin{array}{l}\text { Time of max } \\
\text { firing rate (ms) }\end{array}$ \\
\hline & $38.4 \pm 2.1$ & & $22.8 \pm 1.1$ & \\
\hline & Range: $1.4-229.2$ & $90.0 \pm 1.9$ & Range: $1.2-168.8$ & $68.7 \pm 1.9$ \\
\hline Onset times (ms) & \multicolumn{2}{|c|}{$47.3 \pm 4.2$} & & \\
\hline Offset times (ms) & \multicolumn{2}{|c|}{$138.2 \pm 5.2$} & & \\
\hline
\end{tabular}

This table presents the maximum (Max) firing rates (in spikes/second) and the times (in milliseconds) at which they occurred for reinforced licks (evoked) and unreinforced licks (background), and the range of peak firing rates is also presented. The onset and offset times for the chemosensory responses are also presented. The data are given as the means \pm SEMs.

Table 2. Characteristics of non-chemosensory GC neurons

\begin{tabular}{|c|c|c|c|c|c|c|c|c|c|}
\hline \multicolumn{2}{|c|}{$\begin{array}{l}\text { Reinforced lick, excitatory } \\
(n=26)\end{array}$} & \multicolumn{2}{|c|}{$\begin{array}{l}\text { Reinforced lick, inhibitory } \\
(n=8)\end{array}$} & \multicolumn{2}{|c|}{ Anticipatory $(n=7)$} & \multicolumn{2}{|c|}{$\begin{array}{l}\text { Lick sensitive, tastant modulated } \\
(n=33)\end{array}$} & \multicolumn{2}{|c|}{$\begin{array}{l}\text { Lick sensitive, tastant insensitive } \\
(n=26)\end{array}$} \\
\hline Evoked & Bkg & Evoked & $\mathrm{Bkg}$ & Evoked & Bkg & Evoked & Bkg & Evoked & Bkg \\
\hline \multicolumn{10}{|l|}{ Firing rate (sp/s) } \\
\hline $17.9 \pm 1.2$ & $16.2 \pm 1.2$ & $7.2 \pm 0.7$ & $8.4 \pm 0.8$ & $22.7 \pm 2.0$ & $23.0 \pm 1.9$ & $21.6 \pm 1.6$ & $22.2 \pm 1.7$ & $18.4 \pm 1.1$ & $17.6 \pm 1.0$ \\
\hline \multicolumn{10}{|l|}{ Peak Time (ms) } \\
\hline $82.9 \pm 2.4$ & $68.9 \pm 2.6$ & $62.8 \pm 5.2$ & $75.9 \pm 5.7$ & $-45.0 \pm 5.1$ & $-52.7 \pm 5.8$ & $73.9 \pm 2.5$ & $65.3 \pm 2.3$ & $74.2 \pm 2.8$ & $67.5 \pm 2.7$ \\
\hline
\end{tabular}

This table presents the peak firing rates and times for the evoked and background (Bkg) signals for cells that respond in an excitatory manner to reinforced licking, for cells that inhibit their responses after reinforced licking, anticipatory neurons, tastant-modulated neurons, and strictly lick-sensitive neurons. The conventions are the same as in Table 1.

of termination for the electrode tips is visible. Across all five brains, the electrodes generally spanned layers II-VI, and the electrodes were located primarily in the dysgranular insular cortex. We note that the different types of neuronal responses that are presented below could potentially originate in different cortical layers (Krupa et al., 2004).

\section{Electrophysiological results}

The results are divided into two main sections. The first section details several "types" of evoked responses obtained while rats were engaged in the FR5 schedule. The second section describes how GC neurons responded to changes in concentration and also how the glm was used to fit the chemosensory data and classify the neurons. A total of 178 single units were obtained from five animals, with an average of 35.6 neurons per rat and an average of 1.4 neurons per wire. The numbers of neurons involved in chemosensory, sensorimotor, and/or anticipatory activity are given in Tables 1 and 2.

\section{"Neuronal types"}

Figures 2-4 present examples of responses obtained from rats engaged in the FR5 protocol. The top part of each panel depicts the raster plots, which are sequentially arranged such that the spike train corresponding to the first tastant delivery occurs at the top of the plot. The average activity across all of the deliveries of a particular tastant is depicted in the PSTHs positioned below the raster plots. In these panels, a given tastant was delivered at $0 \mathrm{~s}$ (straight red vertical line). The two dry lick times before and after tastant delivery occurred at about $\pm 150 \mathrm{~ms}$ and are denoted by the red inverted triangles overlaid on the raster plots.

Based on the factors that significantly contributed to the generalized linear model (glm), neurons were classified into different types.

\section{Chemosensory neurons}

Chemosensory neurons were defined as those that discriminated between tastants (including water) as well as discriminating between reinforced and unreinforced licks. Chemosensory neurons comprised $34.3 \%$ (61 of 178 ) of the total neuron population. Figure 2 depicts examples of four different neurons obtained

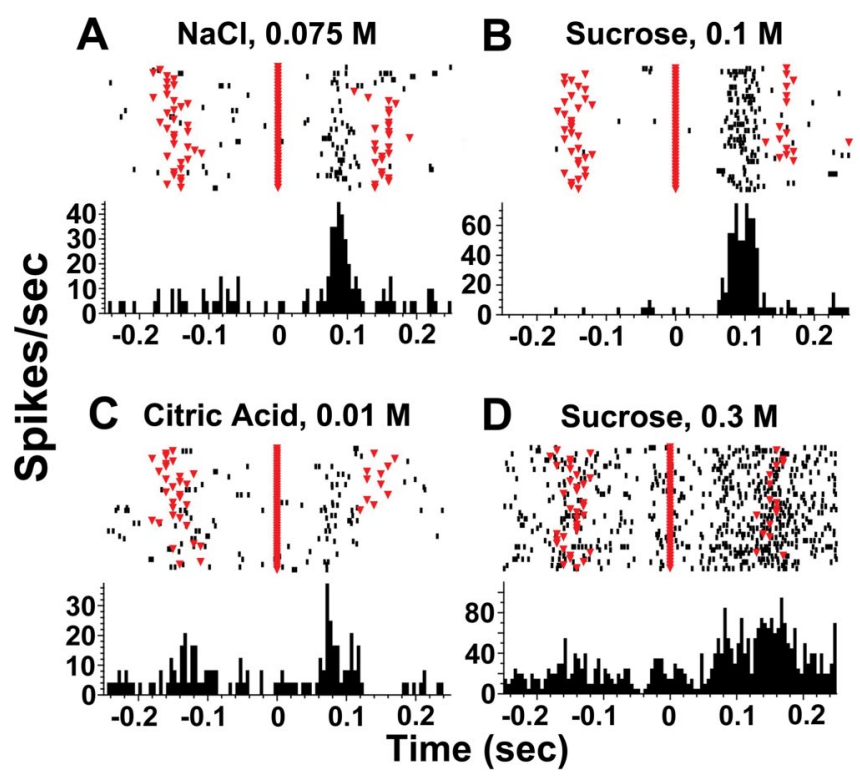

Figure 2. Chemosensitive GC neuronal responses. Shown are examples of four different neurons obtained from the same ensemble. Displayed are the raster plots and PSTHs for the responses of these neurons to multiple stimuli. Zero on the $x$-axis denotes the time of stimulus delivery. The reinforced lick occurs at time 0 (solid red line), and the previous and subsequent licks are visible at around -150 and $150 \mathrm{~ms}$, respectively. These conventions will be followed throughout this paper unless explicitly noted. One outstanding feature of these responses is that their spike trains often exhibit great temporal precision across multiple tastant deliveries $(\boldsymbol{A}-\boldsymbol{C})$ and that the reinforced activity is much greater than the activity for the dry licks. The spike trains of these three neurons displayed a latency of 50-70 ms after tastant delivery, peak response times of $80-90 \mathrm{~ms}$, and offset times of $\sim 100-120 \mathrm{~ms}$. An example of a response to sucrose that continued firing beyond the time of the subsequent lick is shown in $\boldsymbol{D}$.

from the same recorded neural ensemble that reveal characteristics common to nearly all of the chemosensory neurons identified in this study. In these four neurons, the responses to the reinforced licks were greater than those for the unreinforced licks (all values of $\left.p<10^{-23}\right)$. One outstanding feature of these responses was that their spike trains often exhibited great temporal precision across multiple tastant deliveries. In this regard, the spike trains of the neurons shown in Figure 2, $A-C$, displayed a latency of 50-70 ms after tastant delivery, peak response times of $80-90$ 
ms, and offset times of $\sim 100-120 \mathrm{~ms}$ (for population averages, see Table 1). However, as seen in Figure $2 D$, the activity of a small fraction $(8.4 \%$; 15 of 178$)$ of chemosensory neurons continued beyond the time of the subsequent lick. Despite this, the neuron represented in Figure $2 D$ contained sufficient chemosensory information in the first $150 \mathrm{~ms}$ to enable the glm to distinguish among tastants $(p<7 \times$ $10^{-5}$, for all five tastants and their concentrations) (data not shown) as well as between unreinforced and reinforced licks $\left(p<6 \times 10^{-35}\right)$. Because the responses of $91.6 \%$ (153 of 178 ) of the neurons had offset times well before the onset of the lick after the delivery of the tastant (Table 1), and for the other reasons outlined in $\mathrm{Ma}-$ terials and Methods, we have used a 150 ms window to analyze the chemosensory responses obtained under the FR5 schedule.

One additional observation that is clearly evident in these figures is that, after receiving a tastant, the animal may pause before licking again. This was most frequently observed for citric acid (Fig. 2C) and quinine (see Fig. 11), but the same behavior was also exhibited, albeit to a somewhat lesser extent, for other tastants (see Figs. 7, 9).

The responses of a single chemosensory neuron to five different tastants are depicted in Figure 3. This neuron was unresponsive to $0.3 \mathrm{M}$ MSG but responded differentially to the other tastants. The activity corresponding to the reinforced and unreinforced licks was significantly different $\left(p<1 \times 10^{-35}\right)$, and the firing patterns corresponding to different tastants were also different $\left(p<1 \times 10^{-16}\right)$. It is also seen that the firing pattern occurred with great fidelity at specific times. For example, this neuron responded to sucrose on 18 of 24 deliveries with at least one spike between $\sim 90$ and $120 \mathrm{~ms}$. The averaged PSTH for all tastant trials (only five are presented) is shown in the far right panel. A peak at about $-150 \mathrm{~ms}$ in the averaged PSTH corresponds to the fourth unreinforced lick, indicating that this neuron was also lick-sensitive $\left(p<4 \times 10^{-13}\right)$.

The 61 chemosensory neurons were then characterized with regard to their tuning properties as represented by their entropies $(H)$. Of the four chemosensory neurons that were tested with just three tastants (independent of concentration), one neuron responded to three, two responded to two, and one responded to a single tastant. For these four neurons, $H=0.976 \pm 0.013$. Of the eight neurons that were tested with four tastants, one responded to all four and seven responded to two tastants. For these eight neurons, $H=0.988 \pm 0.007$. Of the 50 neurons that were tested with five tastants, 52.9, 25.9, 11.7, 5.9, and $3.9 \%$ responded to five, four, three, two, and one tastant, respectively (Fig. 4A). For these neurons, $H=0.968 \pm 0.009$. In summary, we found GC neurons to be very broadly tuned.

We also calculated to which of the six tastants tested were the chemosensory neurons most responsive. Figure $4 B$ shows the
$\mathrm{NaCl}, 0.3 \mathrm{M}$ Sucrose, $0.3 \mathrm{M}$
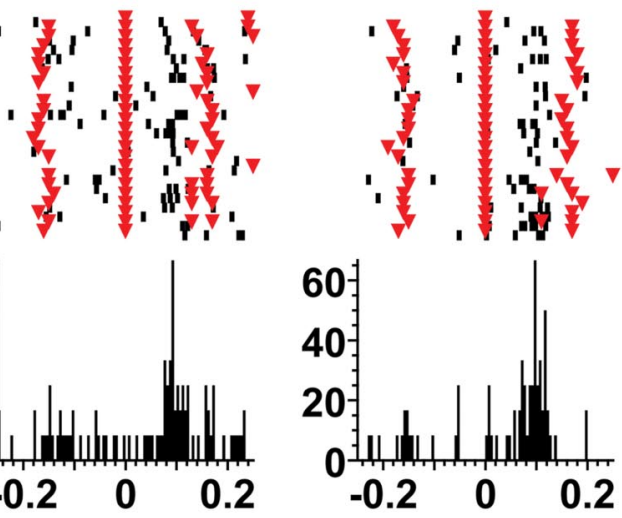

All Taste Trials
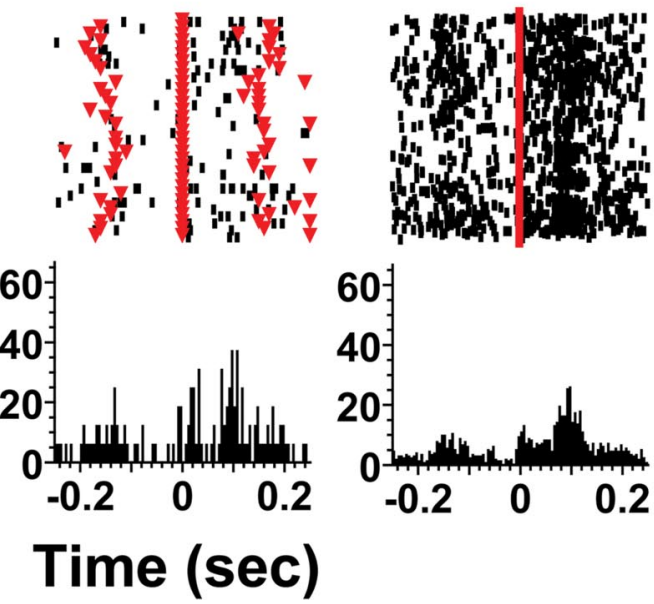

Figure 3. GC neurons exhibit rapid and selective responses to tastants. The activity of this neuron is sufficient to discriminate between reinforced and unreinforced licks as well as between tastants. $\mathrm{NaCl}$ and sucrose (both at $0.3 \mathrm{~m}$ ) elicited the largest it is also broadly tuned. The bottom right panel depicts the responses to all of the tastants across all trials. The unreinforced lick markers have been omitted from this panel for clarity. Symbols are the same as given in Figure 2.
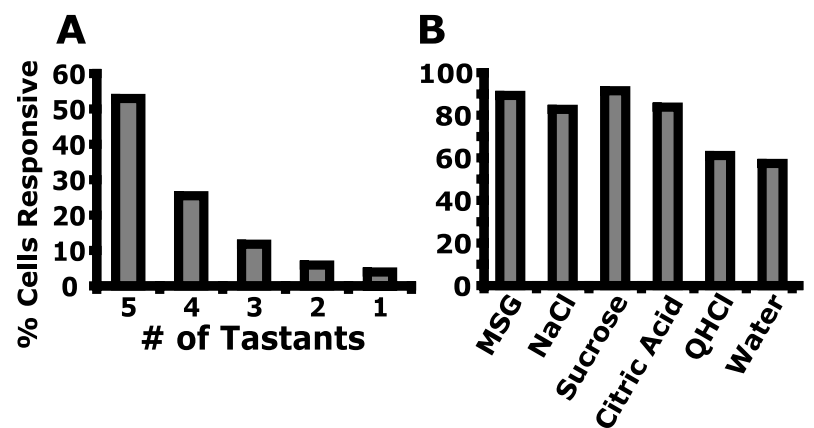

Figure 4. Chemosensory GC neurons are broadly tuned. $\boldsymbol{A}, \mathrm{A}$ histogram showing the percentage of neurons responsive to a given number of tastants. It is seen that most neurons respond to four or five stimuli, indicating that GC neurons are broadly tuned. $\boldsymbol{B}$, This graph depicts the percentage of neurons that responded to each tastant. As seen, most tastants elicit statistically significant responses from gustatory neurons. MSG, NaCl, sucrose, and citric acid elicit responses from approximately the same percentage of neurons, whereas fewer neurons respond to quinine $(\mathrm{QHCl})$ and water.

percentage of chemosensory neurons that responded to at least one concentration of MSG (89.3\%), $\mathrm{NaCl}(82.8 \%)$, sucrose $(91.5 \%)$, citric acid $(83.8 \%)$, quinine $(61.1 \%)$, and water (57.4\%). 


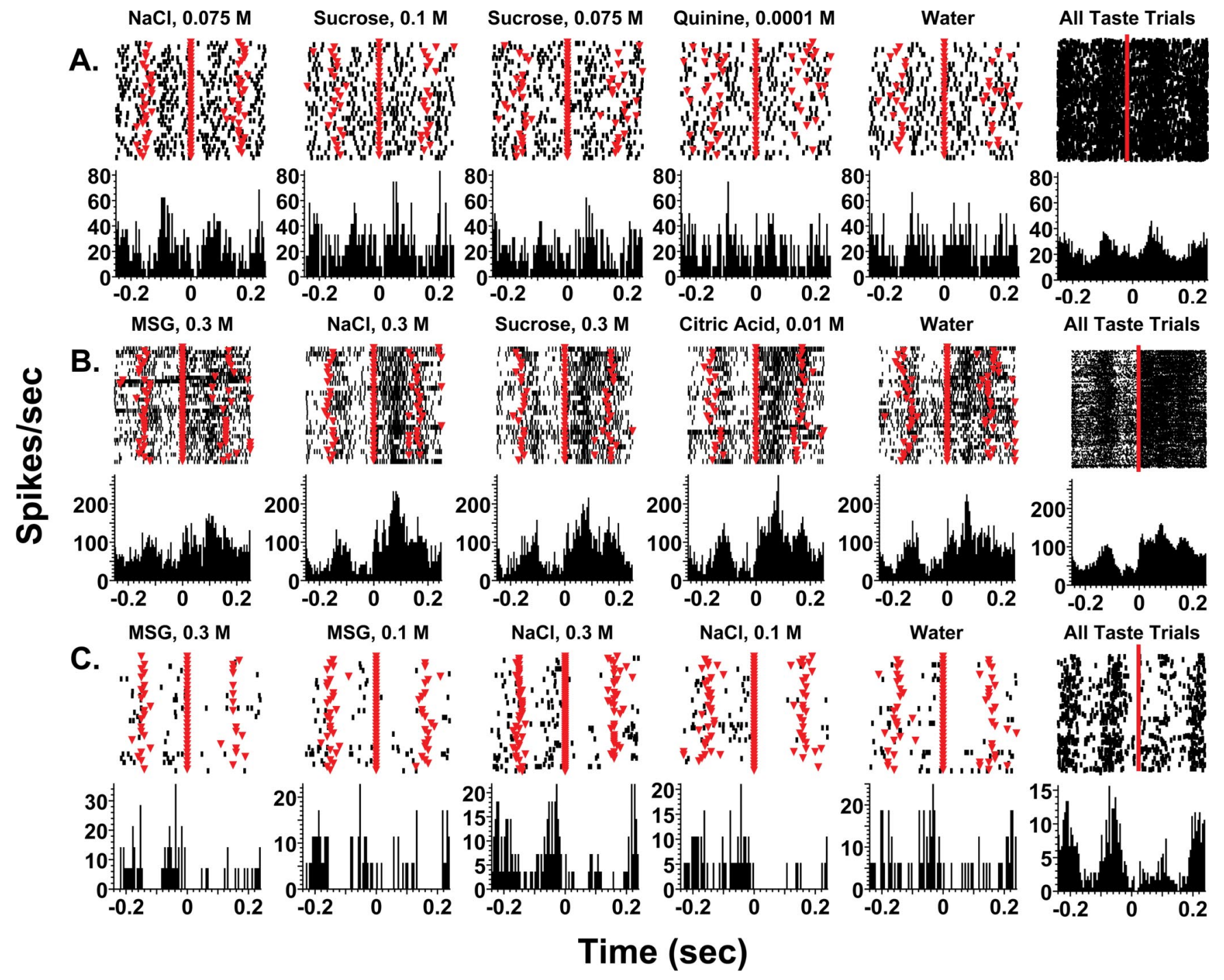

Figure 5. Non-chemosensory neurons that covary with licking. $A$, The evoked activity is shown to occur in oscillatory bursts $50-60 \mathrm{~ms}$ after each lick. This activity, however, is not sufficient to discriminate between reinforced and unreinforced licks $(p>0.4)$ or between tastants $(p>0.8)$. B. This excitatory activity shown was found to be sufficient to discriminate between reinforced and unreinforced licks $(p<0.0001)$, but not between tastants $(p>0.6)$. C, The elicited activity of this neuron was found to be sufficient to discriminate between reinforced and unreinforced licks $(p<$ $\left.4 \times 10^{-20}\right)$, but not between tastants $(p>0.23)$. Here, it is seen that the presence of any tastant causes an inhibition of the firing rate.

To further characterize the firing patterns of these neurons, we calculated the maximum firing rate and the time that it occurred for the tastant-evoked (independent of concentration) and background (third, unreinforced lick) responses. As seen in Table 1, on average, the maximum firing rate was higher in the presence of tastants than for the background $\left(p<8 \times 10^{-13} ; t\right.$ test), whereas the time of this maximum occurred earlier for the background than the evoked response $\left(p<1 \times 10^{-13} ; t\right.$ test $)$. It is on the basis of such differences between firing rate magnitudes and latencies that the glm discriminates between reinforced and unreinforced licks.

Lick-sensitive, tastant-insensitive neurons

Three representative lick-sensitive but tastant-insensitive responses are depicted in Figure 5. For the responses shown in Figure $5 A$, no statistically significant difference was found between reinforced and unreinforced licks $(p>0.4)$ or between tastants $(p>0.8)$. After each lick, the firing rate of this neuron peaked after $\sim 60 \mathrm{~ms}$. In general, for all lick-sensitive, tastantinsensitive neurons, the latency between the time of the lick to the time of peak firing was $74.2 \pm 2.8 \mathrm{~ms}$. Lick-sensitive neurons such as the one shown in Figure 5A comprised 14.6\% (26 of 178) of the population (Table 2).

A second type of lick-sensitive, tastant-insensitive response is shown in Figure 5B. For this example, reinforcement delivery of any tastant tested elicited a strong excitatory response that was greater than the activity evoked by licking the dry tube $(p<$ 0.0001). This neuron responded to all tastants tested with a marked increase in firing at $\sim 80 \mathrm{~ms}$, but failed to discriminate between any of them $(p>0.6)$. This type of neuronal response was found in $14.6 \%$ (26 of 178) of the GC neurons. The averaged PSTH for all tastant trials is depicted in the far right panel. Here, it is evident that a prominent lick-driven response is centered at about $-130 \mathrm{~ms}$. Moreover, it is clear that tastant delivery elicits a much larger response over the background firing rate driven by rhythmic licking. Although the activity of this neuron could rep- 
resent exceptionally broad tuning to tastants, we chose not to classify such neuron types as chemosensory because the glm was unable to discriminate between the tastants.

A third type of lick-sensitive, tastantinsensitive response is depicted in Figure $5 C$. For this neuron, the activity produced by licking a dry tube evoked a phasic excitatory response, but licking for any of the tested tastants resulted in an inhibitory response $\left(p<4 \times 10^{-20}\right)$. An analysis of the posttastant activity revealed an inability to discriminate between any of these tastants $(p>0.23)$. However, because of the low firing rate of this neuron, the tastant-evoked inhibition can be more clearly seen in the averaged PSTH on the extreme right panel where the firing rate of the cell was depressed for $\sim 150 \mathrm{~ms}$ after any of the tastants at different concentrations were delivered. Such fluid-inhibited neurons were less frequently observed $(4.5 \% ; 8$ of 178$)$ than their fluid excited counterparts (Fig. 5B, Table 2).

\section{Tastant-modulated responses}

We also observed neural activity that occurred just before each lick (Fig. 6A). These neurons were termed "anticipatory." The spike trains of this neuron failed to discriminate between reinforced and unreinforced licks $(p>0.3)$, although the firing rate was modulated by the different tastants $\left(p<3 \times 10^{-7}\right)$. Responses of this type $(3.9 \% ; 7$ of 178$)$ displayed very precise temporal fidelity in tracking the onset of each lick.

Still another example of tastantmodulated activity is depicted in Figure $6 B$. Here, it is seen that the activity tracked the licking cycle $\left(p<3 \times 10^{-77}\right)$, but for each tastant the responses for reinforced and unreinforced licks did not differ $(p>$ 0.9). However, the repeated tastant deliveries within the trial caused the overall firing rate of the neuron to increase or decrease $\left(p<9 \times 10^{-15}\right)$. For such tastantmodulated neurons (18.5\%; 33 of 178 ), the background firing rate was different during different tastant trials.

Several characteristics of the nonchemosensory neuronal "types" are presented in Table 2. The time to peak response is the earliest for "anticipatory" neurons at $45.0 \pm 5.1 \mathrm{~ms}$ before reinforced lick, whereas for inhibitory neurons the time to trough is $62.8 \pm 5.2 \mathrm{~ms}$ after reinforced licks. The peaks of the evoked firing rates were greatest for anticipatory neurons $(22.7 \pm 2.0$ spikes/s $)$, although the firing rate for these neurons did not significantly differ from the

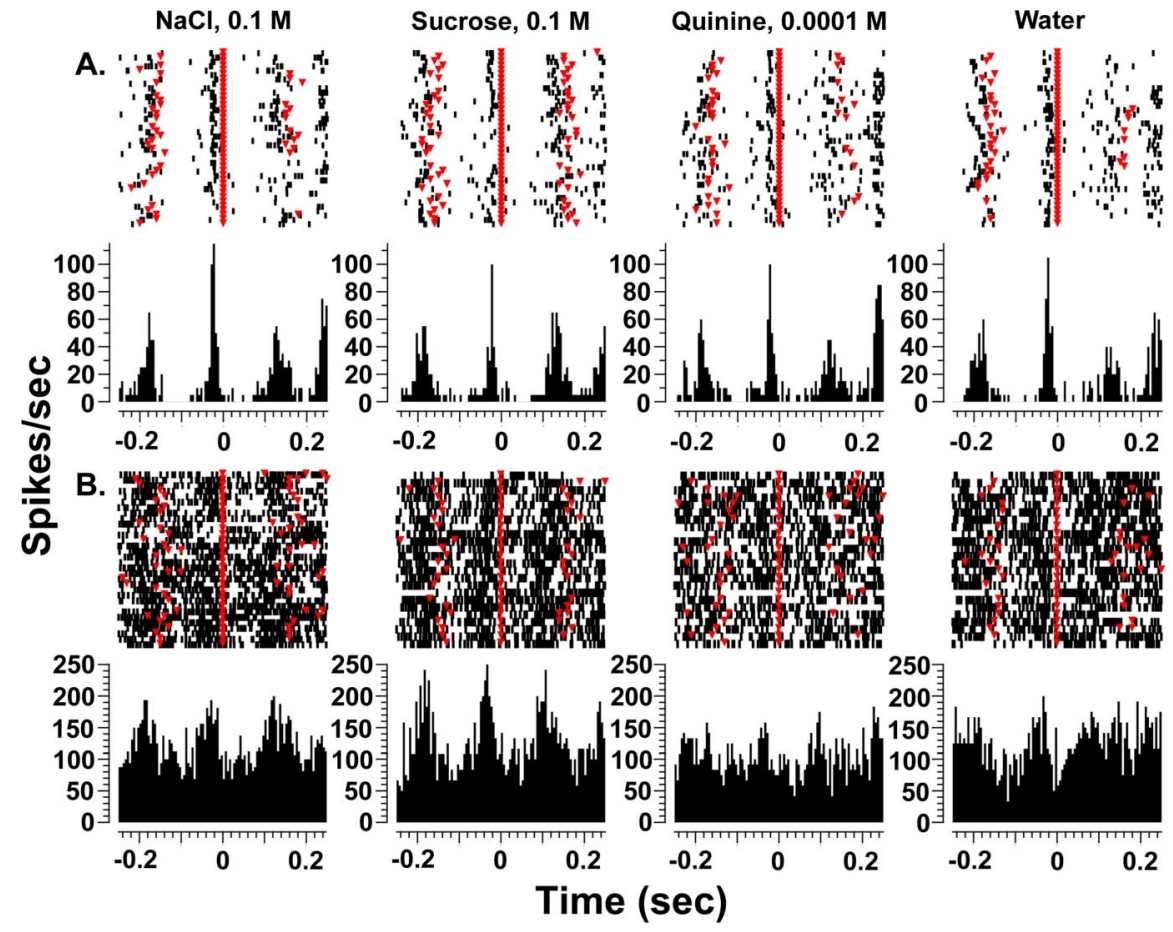

Figure 6. Anticipatory and state-dependent non-chemosensory neurons. $A$, Shown is a response where the neuron rapidly fires $\sim 20$ ms before each lick but fails to discriminate between reinforced and unreinforced licks $(p>0.3)$. Neurons exhibiting this type of activity are termed "anticipatory." B, An example of a neuron whose activity was "state dependent" in that its firing rate was modulated by the presentation of different tastants. The activity of this neuron failed to discriminate between reinforced and unreinforced licks $(p>0.9)$.

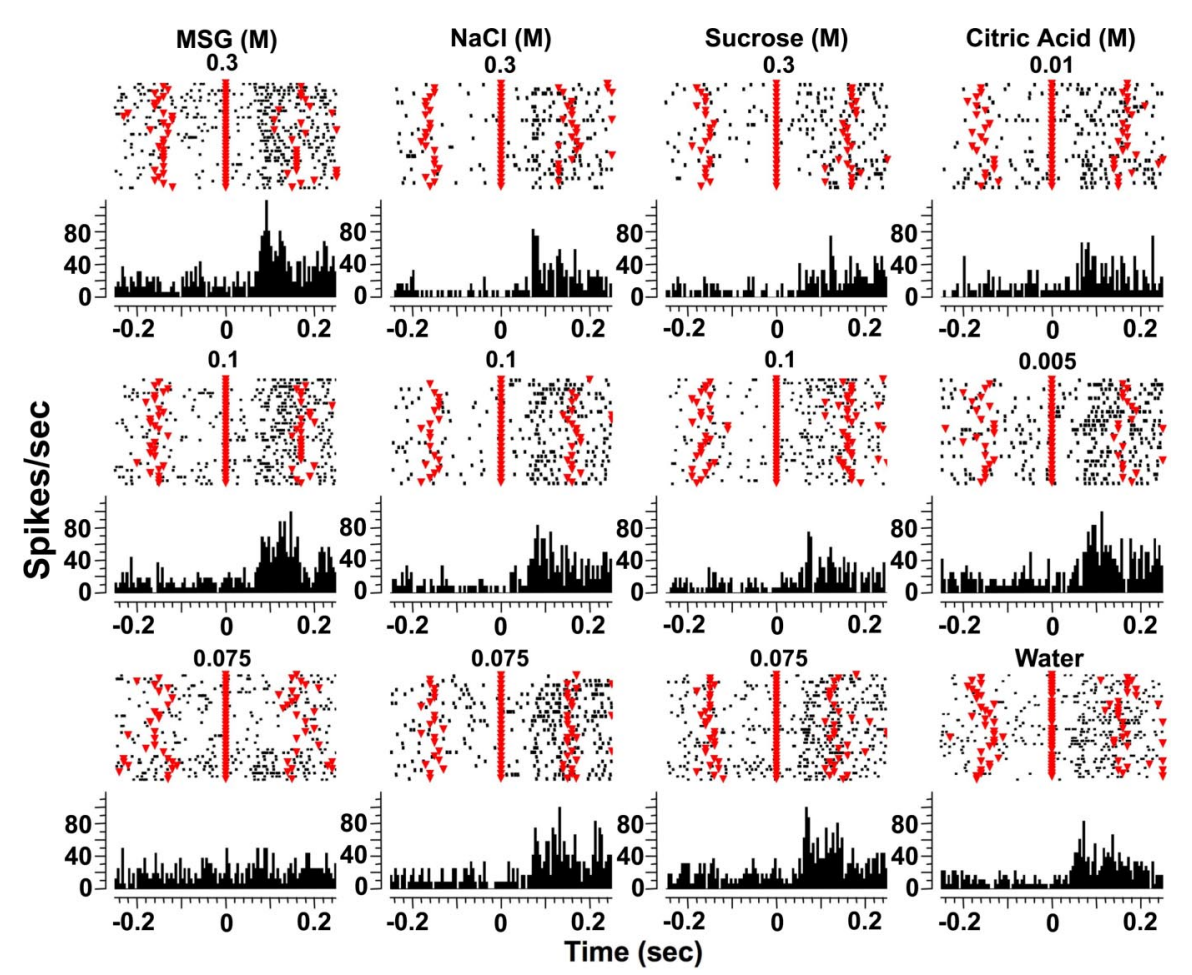

Figure 7. GC neurons exhibit concentration-dependent responses. Shown is the response profile of a single neuron for four tastants at different concentrations and water. This neuron responded to all tastants but was unresponsive to 0.075 m MSG. Robust, temporally precise responses were elicited by 0.3 M MSG and by 0.075 m sucrose. Note also that an increase in MSG concentration results in an increase in firing rate, whereas an increase in sucrose concentration results in a decrease in firing rate. 
tastant-modulated neurons ( $p>0.7 ; t$ test). The evoked firing rates were the least for tastant-inhibited neurons, being $7.2 \pm 0.7$ spikes/s at the trough.

\section{Controls for the influence of expectation}

One concern with using the FR5 protocol is that chemosensory responses potentially can be confounded with activity resulting from the expectation for tastants to be delivered every fifth lick. To test for this possibility, we analyzed the differences between the activities for the second and fourth unreinforced licks. If they differ, then residual tastants might be left in the mouth during lick 2 or anticipation-related responses might develop as the animal perceives that tastants will be available on lick 5 . We found that $55.7 \%$ (34 of 61) of the chemosensory neurons discriminated between the second and fourth unreinforced licks (all values of $p<0.05)$. For only one neuron, the tastant-evoked activity persisted into the second unreinforced lick, so this lick was of greater magnitude than the fourth unreinforced lick (Fig. 2D). In an additional 18 neurons, the second lick was also larger than the fourth lick. For the remaining 15 neurons, the activity evoked by the fourth lick was greater than that evoked by the second lick. However, in all 61 chemosensory neurons, we found that the absolute magnitude of the tastant-evoked response was significantly ( $p \leq 0.05)$ greater than the activity during licking (Table 1; Figs. 2, 3, 7-11; supplemental Figs. S1-S3, available at www. jneurosci.org as supplemental material). Therefore, because the differences between the second and fourth licks are small relative to the magnitude of the tastant-evoked responses, it follows that non-chemosensory contributions do not confound the identification and quantification of chemosensory signals (see Materials and Methods).

In addition, given the temporal complexity of the background and chemosensory responses, we performed another control for possible contributions arising from the animal expecting a tastant to be delivered on the fifth lick. In these experiments, some rats were first tested on the FR5 schedule and then were switched to a variable ratio (VR5) schedule. If the responses seen after tastant delivery are a composite of chemosensory and anticipatory effects, then the responses to tastants should differ between the two schedules. That is, responses on the FR5 schedule might contain both chemosensory and anticipatory components, whereas the VR5 schedule should only elicit chemosensory responses because the animal will not know exactly when the tastants are going to be delivered. If the responses differ under the two schedules, then it is reasonable to assume that these effects are attributable to the expectation of a tastant to be delivered. If, however, the tastant responses remain unchanged between schedules, then the responses that are reported are strictly chemosensory. We found that the responses to tastants for the chemosensory neurons were not significantly different in these two protocols $(p>0.05)$. In the case of the FR5 schedule, subjects might lick in a certain way when they expect to receive hedonically positive tastants on the fifth lick, and might lick another way if they expect to receive hedonically negative tastants. During the VR5 schedule, the animals do not know when the tastant is going to be delivered, so their lick patterns should not vary. Because there are no differences between the tastant responses in FR5 and VR5 schedules, this implies that observed differences in firing patterns are truly chemosensory and are not a product of expectation or different lick patterns for different tastants.

In summary, given that the magnitude of tastant-evoked responses are much greater than the responses during unreinforced licking and that the responses to tastants do not significantly
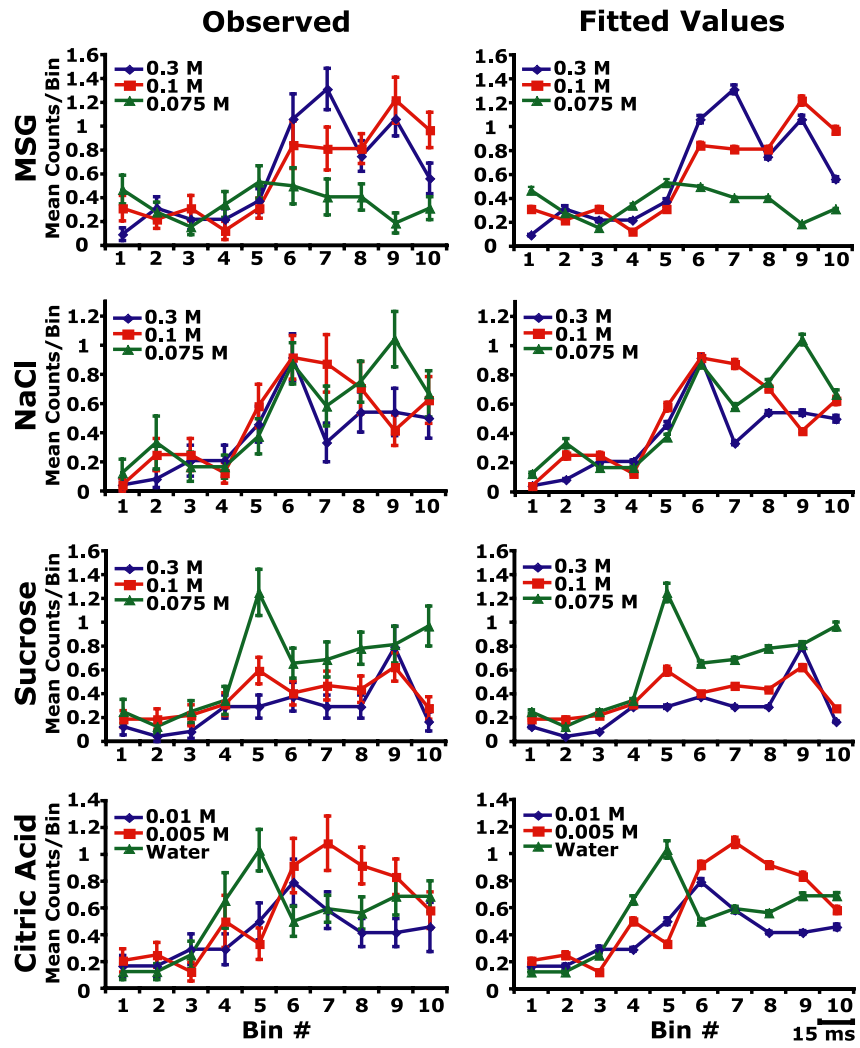

Figure 8. Comparison of evoked responses with the fitted values returned by the glm. The left panels present the observed firing patterns to each of the tastants, and in the right panels the best-fitted values are depicted as given by the $\mathrm{glm}$. The responses to each of tastants are shown over a $150 \mathrm{~ms}$ window, which is the approximate interval between licks. The mean spike counts \pm SEMs for each $15 \mathrm{~ms}$ bin are plotted on the ordinate, and the bin numbers are located on the abscissas. Each row of panels corresponds to the responses of the neuron with respect to different concentrations of a given tastant. The first row of panels corresponds to MSG, the second to $\mathrm{NaCl}$, the third to sucrose, and the fourth to citric acid and water. For each panel, purple diamonds represent the responses to the highest tastant concentration, red squares represent the responses to the middle concentration, and green triangles indicate the responses to the lowest concentration. Note the good agreement between the observed data and the fitted values returned by the glm. The model contains $72.3 \%$ of the observed data within the $90 \%$ predictive interval. As seen in both the observed and fitted data, the time of maximal separation between 0.3 and $0.1 \mathrm{~m} \mathrm{MSG}$ is at $105 \mathrm{~ms}$, whereas the point of maximal separation between $0.075 \mathrm{~m}$ sucrose and the higher concentrations is at $75 \mathrm{~ms}$.

differ between the FR5 and VR5 schedules, it follows that relative to the chemosensory responses the effects of tastant expectation are small and therefore do not confound our quantification of tastant-evoked activity.

\section{Chemical tuning, concentration dependence, and validation of the glm}

The chemical tuning profiles of three chemosensory neurons are presented in Figures 7-12. Figures 7, 9, and 11 depict the raster plots and the PSTHs (with 5 ms bins), and Figures 8, 10, and 12 are presented to demonstrate the capability of the glm to capture many of the temporal characteristics and the tuning profiles of these three neurons (in $15 \mathrm{~ms}$ bins). The complete responses together with the background activity are seen in supplemental Figures S1-S3 (available at www.jneurosci.org as supplemental material). We found that, in the 61 chemosensory neurons, the glm faithfully reconstructed the overall magnitude and dynamics of the PSTHs for each tastant. For these 61 neurons, $85.7 \pm 2.2 \%$ of the observed data fell within the $90 \%$ predictive intervals constructed around the fitted values. This indicates that the predic- 
tions of the glm reasonably represent the original data. The glm, however, does possess limitations. First, it assumes that the $X s$ in different bins are conditionally independent given the $\lambda s$, an assumption which might not be valid. In addition, although the glm accurately reconstructs the overall trends of the data (Figs. 8, 10, 12; supplemental Figs. S1-S3, available at www.jneurosci.

org as supplemental material), it fails to capture all of the small firing rate fluctuations because of the choice of using $15 \mathrm{~ms}$ size bins.

Analysis of the spike trains presented in Figure 7 revealed that they could discriminate between reinforced and unreinforced licks $\left(p<2 \times 10^{-84}\right)$ and between the tastants themselves $\left(p<5 \times 10^{-18}\right)$. This neuron responded to all five tastants tested, although it was unresponsive to 0.075 M MSG $(p>0.07)$. The precise temporal dynamics of the taste responses are evidenced by the response to $0.3 \mathrm{M} \mathrm{MSG}$ that displays a sharp temporal alignment of the onset of activity $80-90 \mathrm{~ms}$ after tastant delivery. This alignment persisted across 32 deliveries of MSG spanning a total of four MSG trials, in which each MSG trial (or 8 deliveries) was separated by 11 trials (88 tastant deliveries) corresponding to the other tastants. Such time-locked responses were similarly robust for $0.075 \mathrm{M}$ $\mathrm{NaCl}, 0.075 \mathrm{M}$ sucrose, and water. This cell also responded to 0.1 $\mathrm{M}$ MSG and to $0.005 \mathrm{M}$ citric acid, but the spike trains were not as time-locked as they were for the higher concentrations of these tastants. The responses to changes in concentration were complex and will be described in detail below.

The mean activity in $15 \mathrm{~ms}$ increments (up to $150 \mathrm{~ms}$ ) for all tastants shown in Figure 7 is depicted in the left panels of Figure 8 , and the fitted values returned by the glm in these same 10 bins are given in the right panels (for clarity, we have omitted here the activity evoked by unreinforced licking; these data are shown in supplemental Fig. S1, available at www.jneurosci.org as supplemental material). This arrangement is also maintained in the remaining two examples [Figs. 10, 12; supplemental Figs. S2, S3 (available at www.jneurosci.org as supplemental material), respectively].

For the activity presented in Figures 7 and 8, the overall fit of the glm to the data are reasonable because $72.3 \%$ of the observed data fell within the $90 \%$ predictive interval. In regard to the example given in Figure 8, it is seen that, for MSG in both the observed and fitted curves, increasing the concentration resulted in an increase in firing rate. Whereas the neuron was unresponsive to $0.075 \mathrm{M} \mathrm{MSG}$, both of the higher concentrations elicited responses (values of $p<2 \times 10^{-10}$ ). For 0.1 and $0.3 \mathrm{M}$ MSG, the two patterns only differ for $15 \mathrm{~ms}$ between 90 and $105 \mathrm{~ms}$. All three $\mathrm{NaCl}$ concentrations elicited responses (values of $p<2 \times$ $10^{-7}$ ), but increasing the $\mathrm{NaCl}$ concentration did not cause the magnitude of the firing rate to increase. Sucrose also elicited significant responses at all concentrations (values of $p<0.004$ ), but the firing rate decreased with increasing concentrations of sucrose. In particular, $0.075 \mathrm{~m}$ sucrose elicited the largest response, and the time of greatest difference between $0.075 \mathrm{M}$ and the other
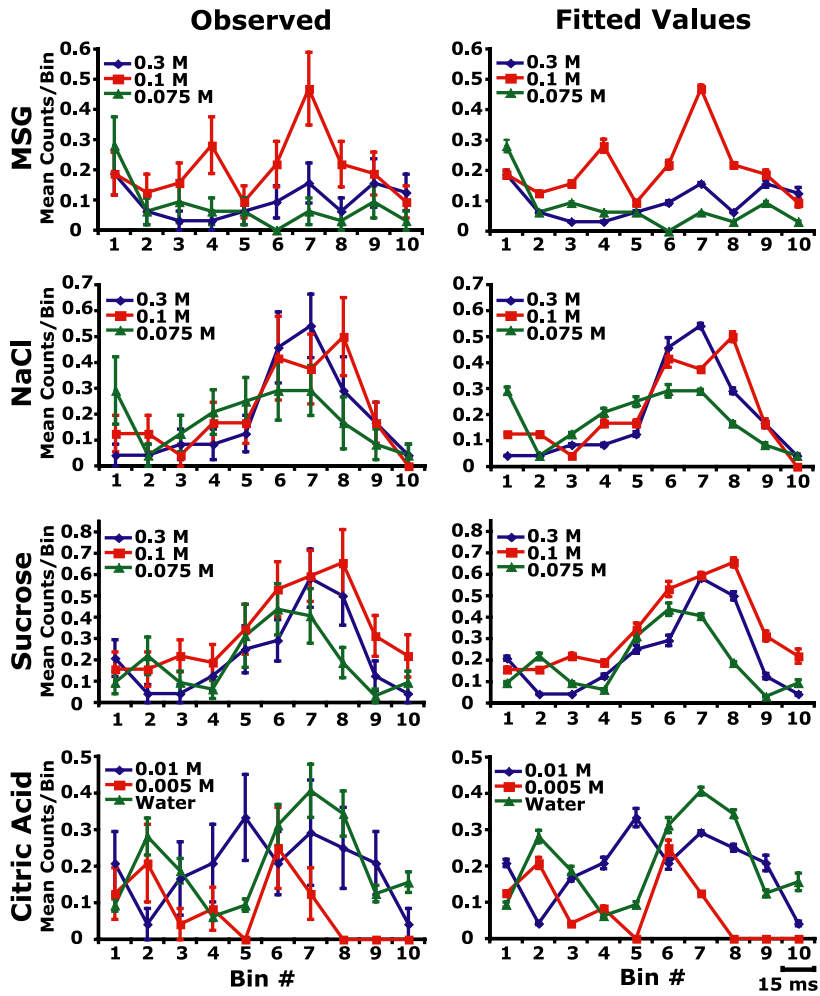

Figure 10. Comparison of observed and fitted values for responses shown in Figure 9. These graphs represent the evolution of the response of the neuron to the tastants over the $150 \mathrm{~ms}$ window. The observed firing patterns are presented in the panels on the left, and the panels on the right depict the best-fit curves generated by the glm. As seen, there is a good overall agreement between the observed data set and the predictions of the model, because $88.5 \%$ of the observed data fell within the $90 \%$ confidence interval. Note that the firing pattern for $0.1 \mathrm{~m}$ MSG differs from 0.075 and $0.3 \mathrm{~m}$ only between 90 and $105 \mathrm{~ms}$. 


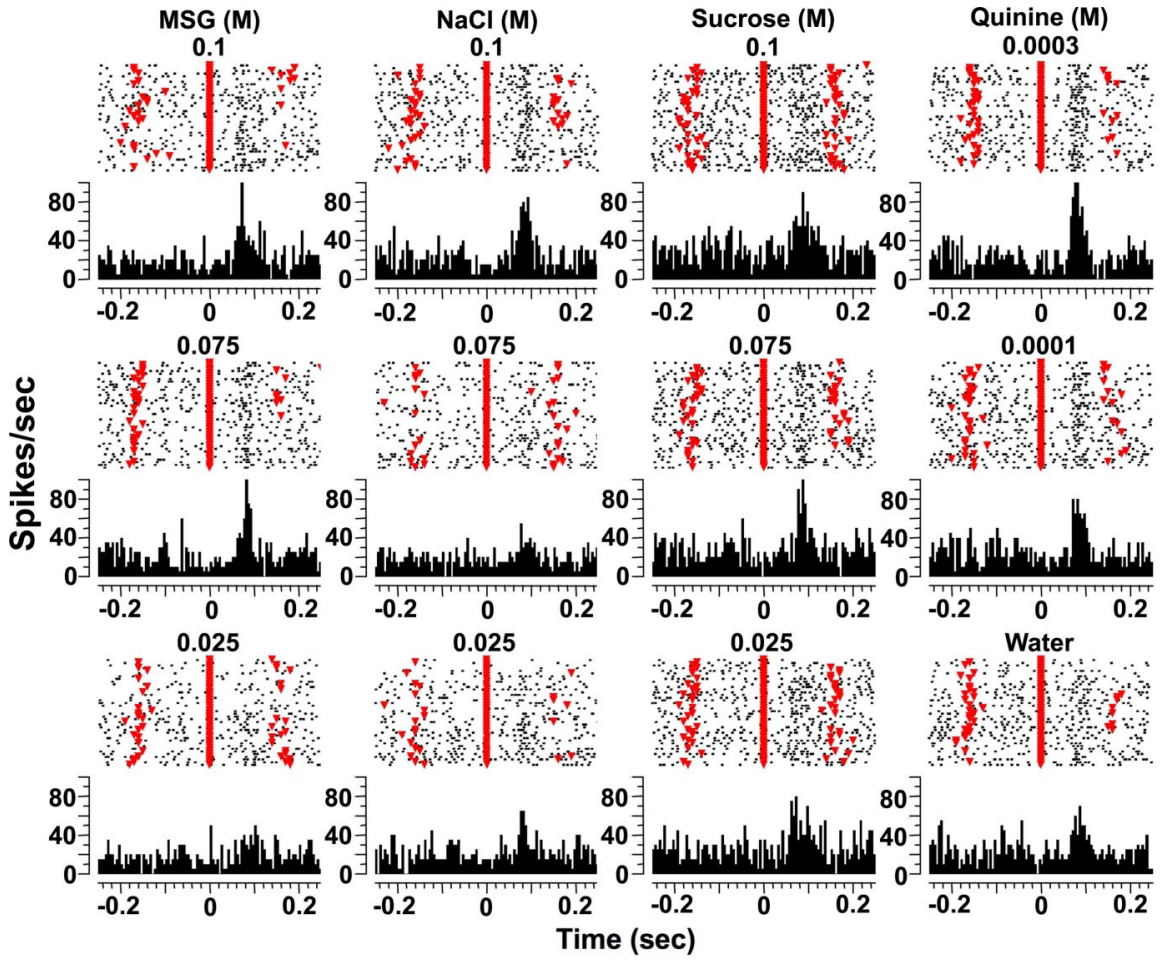

Figure 11. Sucrose and quinine evoke responses in the same GC neuron. Shown are the responses of a neuron to four tastants at multiple concentrations. From the responses, it is evident that this neuron is responsive to both sucrose and quinine. The higher concentration of quinine evokes a larger response than that at $0.0001 \mathrm{~m}$, whereas the intermediate concentration of sucrose elicits the largest response compared with the other concentrations. Note the temporal precision of the firing rate in response to $0.075 \mathrm{M}$ MSG as well as to $0.075 \mathrm{~m}$ sucrose and $0.0003 \mathrm{~m}$ quinine.

two sucrose concentrations is at $75 \mathrm{~ms}$. This neuron also responded to both concentrations of citric acid (values of $p<6 \times$ $\left.10^{-7}\right)$, but the higher concentration elicited a smaller firing rate. Finally, this neuron was also responsive to water $(p<6 \times$ $\left.10^{-14}\right)$. In summary, this example shows that a GC neuron responded to increases in tastant concentration by either increasing or decreasing its firing rates and also by altering its temporal dynamics.

The responses of a different chemosensory neuron in which all five tastants evoked significant phasic activity are presented in Figure 9. The evoked activity differed between reinforced and unreinforced licks $\left(p<1 \times 10^{-35}\right)$ and between tastants $(p<$ $\left.1 \times 10^{-16}\right)$. This example illustrates that selective tastant responses can be evoked by intermediate tastant concentrations. In this regard, of the three MSG concentrations tested, only $0.1 \mathrm{M}$ MSG evoked a response $(p<0.0001)$ with a peak in activity occurring at $105 \mathrm{~ms}$. It is seen that the glm captures the overall shape of the elicited responses (Fig. 10). The responses to $\mathrm{NaCl}$ appeared "normal" because increasing the $\mathrm{NaCl}$ concentration induced increases in firing rates (all values of $p<0.007$ ). For the three $\mathrm{NaCl}$ concentrations, the maximal separation in the patterns occurred at $105 \mathrm{~ms}$. Sucrose evoked responses at all concentrations (values of $p<0.0002$ ) but elicited the most activity at the middle concentration $(0.1 \mathrm{M})$. Only the higher concentration of citric acid $(0.01 \mathrm{M})$ evoked a response $\left(p<2 \times 10^{-5}\right)$ with a peak in activity occurring at $75 \mathrm{~ms}$. Finally, water also evoked a response $\left(p<4 \times 10^{-5}\right)$ with peaks at 30 and $105 \mathrm{~ms}$. For this neuron, $88.5 \%$ of the observed data fell within the $90 \%$ predictive interval constructed by the model.

Figure 11 shows a third example of a broadly tuned neuron that exhibited complex responses to changes in concentration.
This example was chosen to illustrate that GC neurons can respond to both quinine and sucrose, two tastants with diametrically opposed hedonic qualities. The evoked activity was sufficient to discriminate between reinforced and unreinforced licks $\left(p<5 \times 10^{-5}\right)$ and between tastants $\left(p<7 \times 10^{-15}\right)$. Figure 12 displays plots of the activity of this neuron for all tastants in the left panels and the predictions of the model in the right panels. Again, it is seen that the predictions of the model generally match the observed data at every concentration, although some of the small fluctuations are missed (the model captured $63 \%$ of the observed data within the $90 \%$ predictive interval). Although a response to $0.025 \mathrm{M} \mathrm{MSG}$ was not evoked ( $p>$ $0.25)$, robust and temporally precise responses to $0.075 \mathrm{M}$ (75 ms) and 0.1 M MSG (90 ms) were observed (values of $p<$ 0.01 ). The responses to $\mathrm{NaCl}$ were complex in that phasic responses were elicited at 0.1 and $0.025 \mathrm{M}$ (values of $p<0.01$ ), but not at $0.075 \mathrm{M}(p>0.9)$. Responses to sucrose were evoked at all three concentrations tested, but the intermediate concentration $(0.075 \mathrm{M})$ evoked the greatest peak response at $90 \mathrm{~ms}$. This neuron was also activated by quinine, which generated robust, temporally precise responses (peaks $\sim 100 \mathrm{~ms}$ ) that increased with concentration. Finally, water also evoked a response.

Responsiveness of GC neurons to changes in concentration From the three examples given in Figures 7, 9, and 11, it is seen that the GC responses to increases in concentration were not simply a corresponding monotonic increase in firing rate as was found in some (Yaxley et al., 1990; Scott et al., 1991) but not all (Yamamoto et al., 1984) studies. Rather, neurons often exhibited complex responses to changes in concentration. For one tastant, the same neuron might increase its firing rate as the concentration increased, whereas for another tastant it may only be responsive to an intermediate concentration. For the chemosensory neurons, supplemental Table 1 (available at www.jneurosci.org as supplemental material) presents the variety of responses induced by changes in concentration observed for the different tastants. From these data, it is evident that, although a few neurons either monotonically increased or decreased their firing rates in response to changes in concentration of $\mathrm{MSG}, \mathrm{NaCl}$, and sucrose, the vast majority of them $(71.6 \%)$ responded to concentration changes in a complex manner. In such instances, the middle concentration might evoke the highest firing rate (see response to $0.1 \mathrm{M}$ sucrose in Fig. 9), or the highest and lowest concentrations might elicit the highest rates (see $\mathrm{NaCl}$ in Fig. 11), or the neuron might respond to only one concentration (see $0.1 \mathrm{M}$ MSG in Fig. 9). Because citric acid and quinine were tested at only two concentrations, the responses elicited by these tastants could increase, decrease, or remain unchanged. For the case of citric acid and quinine, an approximately equal number of neurons increased or decreased their responses as the concentration increased. In summary, the vast majority of neurons do not re- 
Observed
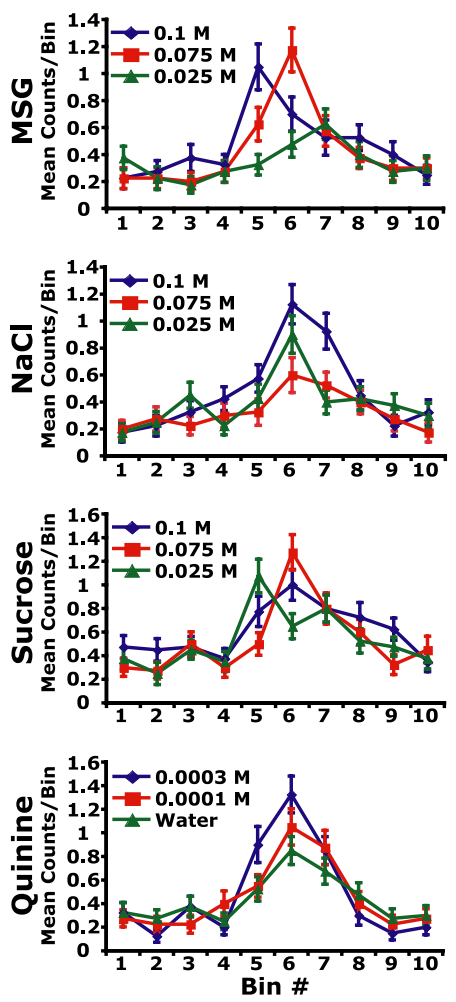

Fitted Values
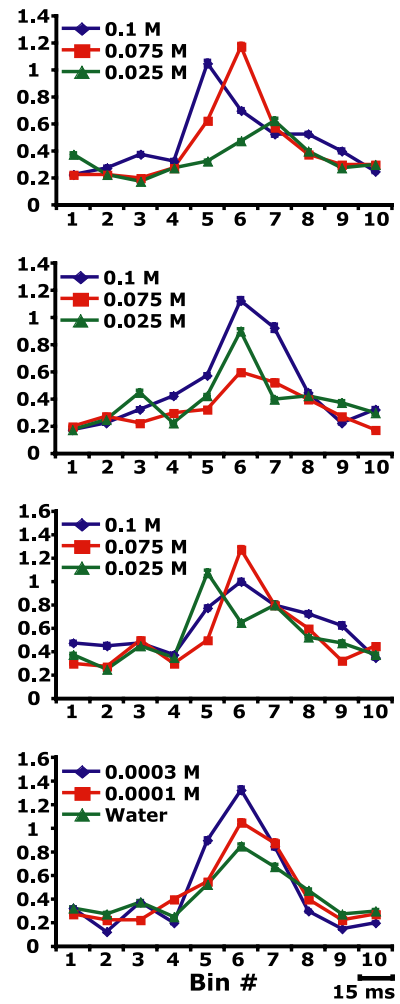

Figure 12. Comparison of observed and fitted values for responses shown in Figure 11. The observed responses of the neuron for each tastant are presented in the graphs on the left, and the right panels depict the fits for each tastant as predicted by the model. The model accounts for $63.0 \%$ of the observed data within the $90 \%$ confidence interval. Note that, for MSG, $0.1 \mathrm{M}$ elicits a peak at $75 \mathrm{~ms}$, whereas $0.075 \mathrm{~m}$ elicits a peak at $90 \mathrm{~ms}$. Hence, a span of $15 \mathrm{~ms}$ can be used to discriminate between concentrations of tastants. Whereas the middle concentration of $\mathrm{NaCl}$ evokes the smallest response, the times to peak after each concentration of $\mathrm{NaCl}$ do not differ among each other. In contrast, different sucrose concentrations elicit peaks at differing times. For $0.025 \mathrm{~m}$ sucrose, the maximal firing rate occurs at $75 \mathrm{~ms}$, whereas for $0.075 \mathrm{~m}$ and for $0.3 \mathrm{M}$, the maximal firing time occurs at $90 \mathrm{~ms}$. Increases in the quinine concentration, however, lead to increases in firing rate without causing the peak response time to shift.

spond monotonically to concentration increases or decreases, but rather they respond in a complex manner.

From the perspective of transduction processes, one would expect the latency of GC responses to be inversely proportional to concentration (Sato et al., 1987; Min et al., 1998). The firing rate latencies were pooled across MSG, $\mathrm{NaCl}$, and sucrose from 0.075 to $0.3 \mathrm{M}$, and this mean was compared by an unpaired $t$ test to the pooled average of the same three tastants at $0.025 \mathrm{M}$. Independent of the tastants, we found that the response times for $0.025 \mathrm{M}$ were significantly longer $(p<0.008)$ than for the response times at
$0.075,0.1$, and $0.3 \mathrm{M}$. Response latencies for tastants at $0.025 \mathrm{M}$ ranged from 97.5 to $120.0 \mathrm{~ms}$, whereas the latencies for the higher concentrations ranged from 78.6 to $97.5 \mathrm{~ms}$ (Table 3). For the higher concentrations, the response times were similar across tastants, whereas the latencies for tastants at $0.025 \mathrm{M}$ are quite significantly different, perhaps because this concentration is closer to threshold and therefore transduction processes will be slower.

\section{Discussion}

Psychophysical studies have shown that rats can identify tastants in a single lick (Halpern and Tapper, 1971). By using a glm to separate somatosensory and gustatory responses, we found that sufficient information is present in the spike trains of GC neurons to distinguish among tastants in a single lick.

\section{Using the glm to analyze GC spike trains}

To determine how chemosensory information may be encoded in recordings from freely licking rats, the contributions of temporally varying somatosensory and chemosensory responses must be separated. We have addressed this problem through the use of a generalized linear model (McCullagh and Nelder, 1989). The fitted values of the glm accord well with the observed data in that $85.7 \%$ of the data for chemosensory neurons fell within the $90 \%$ predictive intervals. In this respect, the glm can better represent precise temporal responses than time-averaged models that use single bins to represent the evoked and background responses (see Materials and Methods). In summary, the glm has been found to be a valuable tool to represent the GC spike trains evoked from freely licking rats.

\section{Lick-sensitive, non-chemosensory neurons}

Multiple electrophysiological studies have indicated that the gustatory cortex in both primates and rodents contains neurons that process combinations of chemosensory, somatosensory, anticipatory, hedonic, and visceral information (Yamamoto et al., 1988, 1989; Yaxley et al., 1990; Plata-Salaman et al., 1992, 1993; Scott et al., 1994, 1999; Katz et al., 2001, 2002; Ogawa and Wang, 2002). These results perhaps should be expected because the GC receives inputs from many cortical areas including the primary somatosensory cortex (SI) (Yoshimura et al., 2003).

Recording from rats engaged in the FR5 schedule revealed several types of neuronal activity. Three non-chemosensory neuronal types covaried with the lick cycle. The first type tracked the onset of each lick with great fidelity but failed to discriminate between reinforced and unreinforced licks (Fig. 5A). Likely, these neurons were either strictly somatosensory or projected to motor nuclei involved in tracking the lick cycle. The second type discriminated between reinforced and unreinforced licks (Fig. 5B) and was likely activated by somatosensory input from fluid in the

Table 3. Peak response times for different concentrations

\begin{tabular}{lclccc}
\hline Molar & MSG & NaCl & Sucrose & Citric acid & Quinine HCl \\
\hline 0.3 & $82.8 \pm 7.5$ & $85.6 \pm 7.2$ & $97.5 \pm 8.0$ & & \\
0.1 & $90.9 \pm 6.5$ & $97.1 \pm 5.0$ & $90.0 \pm 6.1$ & \\
0.075 & $80.0 \pm 6.6$ & $78.6 \pm 6.2$ & $83.6 \pm 7.6$ & \\
0.025 & $120.0 \pm 11.3$ & $97.5 \pm 20.7$ & $103.9 \pm 9.7$ & & \\
0.01 & & & & $87.0 \pm 5.3$ &
\end{tabular}


oral cavity that was absent in unreinforced licks (Rolls et al., 2003; Verhagen et al., 2003). The third type also differentiated between reinforced and unreinforced licks, but the delivery of any tastant resulted in an inhibition of responses (Fig. 5C). This type of neuronal response, which was also seen in the SI of freely whisking rats (Krupa et al., 2004), could contribute to encoding the presence of fluid in the mouth.

Two additional types of non-chemosensory responses were also identified (Fig. 6). The first exhibited a temporally precise and sparse response that occurred immediately before the onset of each lick. Such a response could represent the activity related to jaw opening or tongue protrusion (Travers et al., 1997). The next type of lick-sensitive response was one in which the neuron failed to discriminate between reinforced and unreinforced licks but for which the overall firing rate was modulated by the different tastants (Fig. 6B). This activity appears to be "state dependent," because these responses may be a consequence of the animals learning that the tastants were delivered in blocks of eight and hence had differing expectation levels during different tastant trials. This translates into an overall firing rate modulation that may reflect the way the subjects track different tastant trials.

\section{Chemosensory neurons}

We found that $34.2 \%$ of the GC neurons exhibited chemosensory responses. These neurons were very broadly tuned, possessing an average entropy of $H=0.971 \pm 0.007(n=61)$. Previous single unit recordings from GC in awake or anesthetized rats have found average entropy values of 0.54 (Yamamoto et al., 1984, 1989), which approximately matches the 0.56-0.75 found in awake, nonhuman primates (Yaxley et al., 1990; Scott et al., 1991; Smith-Swintosky et al., 1991). That our entropies were higher than those previously reported can be rationalized, in part, by noting that the gustatory responses often differ significantly from baseline for only a few milliseconds (Figs. 2, 3, 7, 9, 11), and averaging the activity over several seconds would eradicate this temporally precise information. Another reason is that, for each tastant, the maximal response evoked at any concentration was selected for the calculation. That these neurons are broadly tuned indicates that any gustatory coding scheme should, to a large extent, be combinatorial (Ganchrow and Erickson, 1970).

Another implication of the broad tuning profile of GC neurons involves the spatial separation of taste responses. In this regard, of the 18 chemosensory neurons that were tested with both sucrose and quinine, 11 responded to both stimuli, and of the 56 that were tested with both $\mathrm{NaCl}$ and sucrose, $76.8 \%$ responded to both. This is of interest because receptors for sweet and bitter tastants are found on distinct populations of taste receptor cells that, when activated, lead to predictable behaviors of acceptance or rejection (Zhang et al., 2003). In addition, a recent morphological study found that the mouse gustatory pathway, including the GC, maintains separate populations of neurons for bitter and sweet tastants (Sugita and Shiba, 2005). Additionally, intrinsic imaging studies of rat $\mathrm{GC}$ have indicated that $\mathrm{NaCl}$ and sucrose elicit activity in separate spatial locations (Yoshimura et al., 2004). Other electrophysiological studies of both rat and nonhuman primate GC, however, have indicated that neurons can respond to both sucrose and quinine and to sucrose and $\mathrm{NaCl}$ (Scott et al., 1994; Katz et al., 2001). At this time, the origin of this discrepancy is unclear. However, it may be partially rationalized by noting that electrophysiological recordings can detect smaller changes than do morphological studies and/or that the neurons from which we have recorded may not be those connected to the apparent labeled line from the taste cells.

\section{Coding}

Throughout the gustatory pathway, there have been numerous studies of the effects of changes in the tastant concentration on responses to chemical stimuli. Studies with either anesthetized or awake rodents or nonhuman primates have shown that increasing the tastant concentration will usually produce increases in firing rates. This would be expected if the firing rates were related to the perceptual intensity of the tastants as was found in GC responses in nonhuman primates (Yaxley et al., 1990; Scott et al., 1991). Accordingly, we found responses that increased with tastant concentration (Figs. 7, 8). However, both Yamamoto et al. (1984) and we found that, in response to increases in concentration, not all chemosensitive GC neurons increase their firing rates. Moreover, in the same neuron, the responses to one tastant may increase with concentration, whereas they may decrease for another and for a third tastant a middle concentration may elicit the greatest activity (Figs. 11, 12). Indeed, as presented in supplemental Table 1 (available at www.jneurosci.org as supplemental material), the majority of neurons respond to concentration changes with complex, nonlinear changes in firing rate. In summary, changes in concentration can result in a variety of GC responses that reflect both changes in firing rates and changes in the dynamics of the response. We suggest that both properties may be used to encode changes in concentration. We speculate that, in response to concentration changes, such varied behavior could be a consequence of the fact that GC receives (and transmits) information from other cortical and subcortical areas (Smith and Li, 2000; Lundy and Norgren, 2004) and that these areas will respond differentially to different tastants and concentrations of tastants. In particular, tastants such as quinine and sucrose possess different hedonic valences, so additional networks may be recruited to reflect different palatabilities (Small et al., 2003; Sewards, 2004).

\section{Statistically sufficient information to discriminate among tastants is available in $150 \mathrm{~ms}$}

Behavioral studies have shown that rats can discriminate among tastants in a single lick (Halpern and Tapper, 1971). In the visual and auditory systems, the minimum amount of time required by human subjects to perceive a stimulus is $\sim 150-200 \mathrm{~ms}$ (Koch, 2005). In the olfactory system, rats are capable of discriminating between odorants in a single sniff, or $<200 \mathrm{~ms}$ (Uchida and Mainen, 2003). Together, it appears that, in many sensory systems, $\sim 150 \mathrm{~ms}$ is sufficient time for organisms to distinguish among stimuli. Here, we have shown, for the first time, that GC recordings from motivated animals contain sufficient information to discriminate among tastants in a single lick. Approximately $70 \mathrm{~ms}$ after tastant delivery, statistically significant chemosensory differences could be discriminated in the subsequent 50-80 ms within the 150 ms lick cycle (Figs. 7-12; supplemental Figs. S1-S3, available at www.jneurosci.org as supplemental material). This suggests that, in a motivated, freely behaving animal, salient chemosensory information sufficient to distinguish among stimuli can occur in very short time intervals. Indeed, it is likely that it is the very precise temporal responses to tastants that permit this discrimination. This feature suggests that temporal dynamics may also be an important component in the processing of taste-related information (Katz et al., 2002; Di Lorenzo et al., 2003; Katz, 2003).

\section{References}

Bahar A, Dudai Y, Ahissar E (2004) Neural signature of taste familiarity in the gustatory cortex of the freely behaving rat. J Neurophysiol 92:3298-3308. 
Berridge KC, Fentress JC (1985) Trigeminal-taste interaction in palatability processing. Science 228:747-750.

Cerf-Ducastel B, Van de Moortele PF, MacLeod P, Le Bihan D, Faurion A (2001) Interaction of gustatory and lingual somatosensory perceptions at the cortical level in the human: a functional magnetic resonance imaging study. Chem Senses 26:371-383.

de Araujo IE, Rolls ET (2004) Representation in the human brain of food texture and oral fat. J Neurosci 24:3086-3093.

de Araujo IE, Kringelbach ML, Rolls ET, McGlone F (2003) Human cortical responses to water in the mouth, and the effects of thirst. J Neurophysiol 90:1865-1876.

Delay ER, Beaver AJ, Wagner KA, Stapleton JR, Harbaugh JO, Catron KD, Roper SD (2000) Taste preference synergy between glutamate receptor agonists and inosine monophosphate in rats. Chem Senses 25:507-515.

Di Lorenzo PM, Hallock RM, Kennedy DP (2003) Temporal coding of sensation: mimicking taste quality with electrical stimulation of the brain. Behav Neurosci 117:1423-1433.

Ganchrow JR, Erickson RP (1970) Neural correlates of gustatory intensity and quality. J Neurophysiol 33:768-783.

Grimmett G, Stirzaker D (2001) Probability and random processes, Ed 3. Oxford: Oxford UP.

Gutierrez R, Carmena JM, Nicolelis MAL, Simon SA (2006) Orbitofrontal ensemble activity monitors licking and distinguishes among natural rewards. J Neurophysiol 95:119-133.

Halpern BP, Tapper DN (1971) Taste stimuli: quality coding time. Science $171: 1256-1258$

Katz DB (2003) Making time with taste. Focus on "Taste response variability and temporal coding in the nucleus of the solitary tract of the rat." J Neurophysiol 90:1375-1376.

Katz DB, Simon SA, Nicolelis MA (2001) Dynamic and multimodal responses of gustatory cortical neurons in awake rats. J Neurosci 21:4478-4489.

Katz DB, Simon SA, Nicolelis MA (2002) Taste-specific neuronal ensembles in the gustatory cortex of awake rats. J Neurosci 22:1850-1857.

Koch C (2005) The movie in your head. Sci Am Mind 16:58-63.

Kosar E, Grill HJ, Norgren R (1986) Gustatory cortex in the rat. I. Physiological properties and cytoarchitecture. Brain Res 379:329-341.

Krupa DJ, Wiest MC, Shuler MG, Laubach M, Nicolelis MAL (2004) Layerspecific somatosensory cortical activation during active tactile discrimination. Science 304:1989-1992.

Lundy Jr RF, Norgren R (2004) Activity in the hypothalamus, amygdala, and cortex generates bilateral and convergent modulation of pontine gustatory neurons. J Neurophysiol 91:1143-1157.

Lyall V, Heck GL, DeSimone JA, Feldman GM (1999) Effects of osmolarity on taste receptor cell size and function. Am J Physiol 277:C800-C813.

Lyall V, Heck GL, Phan TH, Mummalaneni S, Malik SA, Vinnikova AK, DeSimone JA (2005) Ethanol modulates the VR-1 variant amilorideinsensitive salt taste receptor. I. Effect on TRC volume and $\mathrm{Na}^{+}$flux. J Gen Physiol 125:569-585.

McCullagh P, Nelder JA (1989) Generalized linear models, Ed 2. London: Chapman and Hall.

Meck WH (2003) Functional and neural mechanisms of interval timing. Boca Raton, FL: CRC.

Min BC, Wada M, Sakamoto K (1998) New apparatus stimulating the region of tongue innervated by glossopharyngeal nerve and its application to monosodium glutamate (MSG) solution. Appl Hum Sci 17:67-71.

Ogawa H, Wang XD (2002) Neurons in the cortical taste area receive nociceptive inputs from the whole body as well as the oral cavity in the rat. Neurosci Lett 322:87-90.

Ogawa H, Murayama N, Hasegawa K (1992) Difference in receptive field features of taste neurons in rat granular and dysgranular insular cortices. Exp Brain Res 91:408-414.

Plata-Salaman CR, Scott TR, Smith-Swintosky VL (1992) Gustatory neural coding in the monkey cortex: L-amino acids. J Neurophysiol 67:1552-1561.

Plata-Salaman CR, Scott TR, Smith-Swintosky VL (1993) Gustatory neural coding in the monkey cortex: the quality of sweetness. J Neurophysiol 69:482-493.

Plata-Salaman CR, Scott TR, Smith-Swintosky VL (1995) Gustatory neural coding in the monkey cortex: acid stimuli. J Neurophysiol 74:556-564.

Rolls ET, Verhagen JV, Kadohisa M (2003) Representations of the texture of food in the primate orbitofrontal cortex: neurons responding to viscosity, grittiness, and capsaicin. J Neurophysiol 90:3711-3724.
Sato T, Miyamoto T, Okada Y (1987) Latency of gustatory neural impulses initiated in frog tongue. Brain Res 424:333-342.

Scott TR, Plata-Salaman CR, Smith VL, Giza BK (1991) Gustatory neural coding in the monkey cortex: stimulus intensity. J Neurophysiol 65:76-86.

Scott TR, Plata-Salaman CR, Smith-Swintosky VL (1994) Gustatory neural coding in the monkey cortex: the quality of saltiness. J Neurophysiol 71:1692-1701.

Scott TR, Giza BK, Yan J (1999) Gustatory neural coding in the cortex of the alert cynomolgus macaque: the quality of bitterness. J Neurophysiol 81:60-71.

Sewards TV (2004) Dual separate pathways for sensory and hedonic aspects of taste. Brain Res Bull 62:271-283.

Smith D, Travers JB (1979) A metric for the breadth of tuning of gustatory neurons. Chem Senses Flav 4:215-229.

Small DM, Gregory MD, Mak YE, Gitelman D, Mesulam MM, Parrish T (2003) Dissociation of neural representation of intensity and affective valuation in human gustation. Neuron 39:701-711.

Small DM, Voss J, Mak YE, Simmons KB, Parrish T, Gitelman D (2004) Experience-dependent neural integration of taste and smell in the human brain. J Neurophysiol 92:1892-1903.

Smith DV, Li CS (2000) GABA-mediated corticofugal inhibition of tasteresponsive neurons in the nucleus of the solitary tract. Brain Res 858:408-415

Smith-Swintosky VL, Plata-Salaman CR, Scott TR (1991) Gustatory neural coding in the monkey cortex: stimulus quality. J Neurophysiol 66:1156-1165.

Spector AC, St John SJ (1998) Role of taste in the microstructure of quinine ingestion by rats. Am J Physiol 274:R1687-R1703.

Spector AC, Redman R, Garcea M (1996) The consequences of gustatory nerve transection on taste-guided licking of sucrose and maltose in the rat. Behav Neurosci 110:1096-1109.

Stapleton JR, Luellig M, Roper SD, Delay ER (2002) Discrimination between the tastes of sucrose and monosodium glutamate in rats. Chem Senses 27:375-382.

Sugita M, Shiba Y (2005) Genetic tracing shows segregation of taste neuronal circuitries for bitter and sweet. Science 309:781-785.

Todrank J, Bartoshuk LM (1991) A taste illusion: taste sensation localized by touch. Physiol Behav 50:1027-1031.

Travers JB, Dinardo LA, Karimnamazi H (1997) Motor and premotor mechanisms of licking. Neurosci Biobehav Rev 21:631-647.

Uchida N, Mainen ZF (2003) Speed and accuracy of olfactory discrimination in the rat. Nat Neurosci 6:1224-1229.

Verhagen JV, Rolls ET, Kadohisa M (2003) Neurons in the primate orbitofrontal cortex respond to fat texture independently of viscosity. J Neurophysiol 90:1514-1525.

Wiesenfeld Z, Halpern BP, Tapper DN (1977) Licking behavior: evidence of hypoglossal oscillator. Science 196:1122-1124.

Yamamoto T, Matsuo R, Kawamura Y (1980) Localization of cortical gustatory area in rats and its role in taste discrimination. J Neurophysiol 44:440-455.

Yamamoto T, Yuyama N, Kato T, Kawamura Y (1984) Gustatory responses of cortical neurons in rats. I. Response characteristics. J Neurophysiol 51:616-635.

Yamamoto T, Matsuo R, Kiyomitsu Y, Kitamura R (1988) Sensory inputs from the oral region to the cerebral cortex in behaving rats: an analysis of unit responses in cortical somatosensory and taste areas during ingestive behavior. J Neurophysiol 60:1303-1321.

Yamamoto T, Matsuo R, Kiyomitsu Y, Kitamura R (1989) Taste responses of cortical neurons in freely ingesting rats. J Neurophysiol 61:1244-1258

Yaxley S, Rolls ET, Sienkiewicz ZJ (1990) Gustatory responses of single neurons in the insula of the macaque monkey. J Neurophysiol 63:689-700.

Yoshimura H, Kato N, Sugai T, Segami N, Onoda N (2003) Age-dependent appearance of an insulo-parietal cortical signal propagation that elicits a synchronized population oscillation in the parietal cortex in rats. Brain Res Dev Brain Res 143:245-251.

Yoshimura H, Sugai T, Fukuda M, Segami N, Onoda N (2004) Cortical spatial aspects of optical intrinsic signals in response to sucrose and $\mathrm{NaCl}$ stimuli. NeuroReport 15:17-20.

Zeigler HP, Jacquin MF, Miller MG (1984) Trigeminal sensorimotor mechanisms and ingestive behavior. Neurosci Biobehav Rev 8:415-423.

Zhang Y, Hoon MA, Chandrashekar J, Mueller KL, Cook B, Wu D, Zuker CS, Ryba NJ (2003) Coding of sweet, bitter, and umami tastes: different receptor cells sharing similar signaling pathways. Cell 112:293-301. 